\title{
The Effects of the Charge Density and Structure of the Polymer on the Dye-Binding Characteristics of Some Cationic Polyelectrolytes
}

\author{
B. VISHALAKSHI \\ Department of Inorganic and Physical Chemistry, Indian Institute of Science, Bangalore-560 012, India
}

\begin{abstract}
SYNOPSIS
The interaction of various structurally different water-soluble cationic polyelectrolytes with methyl orange, an anionic dye, has been studied. The changes in the UV-Vis spectra of methyl orange in the presence of methyl and benzyl quaternized polyvinylpyridines and trialkylammonium and triphenylphosphonium salts of polyvinylbenzyl chloride have been used to investigate the effect of the structure on the dye-binding characteristics of the polycations. The results are interpreted in the light of a simple theory of cooperative binding to a linear lattice. The extent of binding of the dye aggregates on the polymers has been calculated. The binding ability of the above-mentioned polyelectrolytes has been found to depend on the nature of the substituent on the polycation. The contribution of the coulombic forces on binding of the dye by these polymers has been emphasized. (C) 1995 John Wiley \& Sons, Inc.
\end{abstract}

Keywords: methyl orange - polycation - quaternization - polyvinylbenzyl chloride • polyvinylpyridine $\bullet$ stacking $\bullet$ cooperativity

\section{INTRODUCTION}

The studies on the interaction of synthetic polymers with ionic dye molecules originated from the studies on the binding of small molecules by proteins. ${ }^{1}$ Such investigations have provided useful information about the nature and mechanism of the binding process. It is evident that both nonpolar and electrostatic interactions are involved in the binding of dye molecules on the polymers. ${ }^{2-4}$

The interaction between the anionic dye molecules and the water-soluble cationic polyelectrolytes has been studied on several systems, taking methyl orange (MO) and its homologs as the dye components. ${ }^{5}$ The synthetic polycations studied include polyethyleneimine,${ }^{1,2,6}$ derivatives of polyvinylimidazole $^{3,4}$ and polyvinylpyridine, ${ }^{7-9}$ polyvinylbenzyltriethylammonium chloride, ${ }^{7}$ polydimethyldiallylammonium chloride, ${ }^{10,11}$ etc. It has been observed

Journal of Polymer Science: Part A: Polymer Chemistry, Vol. 33, 365-371 (1995) (C) 1995 John Wiley \& Sons, Inc that the changes in the UV-Vis spectrum of the dye in the presence of these polymers are due to the binding of the dye molecules on the polycations. The effect is cooperative under certain conditions and is due to the bound dye-dye interactions, which are associated with the aggregation tendency of the dye molecules.

Earlier studies of the spectral behavior of $\mathrm{MO}$ in the aqueous media ${ }^{7}$ show that the dye does not selfaggregate in concentrations as high as $10^{-3} \mathrm{M}$, and its high extinction coefficient makes it possible to use a dye concentration as low as $10^{-5} \mathrm{M}$. For the aggragated state, which is formed in the presence of the polycation, the UV-Vis spectra shows a substantial blue shift. Hence, in this work, we have used MO to probe the dye-binding characteristics of various polycations of different structure with regard to the steric hindrance at the charged site, the nature of the pendant chain, and the charge density of the polymer. Trialkylammonium and triphenylphosphonium salts of polyvinylbenzylchloride and polyvinylpyridine quaternized with alkyl and aryl halides have been selected for the present study. 


\section{EXPERIMENTAL}

The dye, MO, was obtained from BDH chemicals and was purified by recrystallization from ethanol. Polyvinylbenzyl chloride (PVBC) was obtained from Aldrich Chemicals and the chloroform soluble portion was used. The degree of chloromethylation was 1.0 as determined by ${ }^{1} \mathrm{H}-\mathrm{NMR}(200 \mathrm{MHz}$, Bruker). 2-vinylpyridine (2VP) and 4-vinylpyridine (4VP) were obtained from Merck and were distilled twice under reduced pressure before use. The preparations of the corresponding polymers and their methyl derivatives have been reported elsewhere. ${ }^{12}$ All other chemicals were reagent grade and were used as received. The structure of the polymers used in this study are shown in Table I.

\section{Polymer Synthesis}

\section{Poly (vinylbenzyltrimethylammonium chloride) $\left(_{\text {PVBTMAC) }}{ }^{13}\right.$}

PVBC ( $1.0 \mathrm{~g}, 6.56 \mathrm{mmol})$ in dioxane $(20 \mathrm{~mL})$ and trimethylamine $(7.2 \mathrm{~mL}, 40 \mathrm{mmol})$ were stirred at room temperature. The product precipitates as amination proceeds. Minimum quantity of water was then added to redissolve the product and the reaction was allowed to proceed for $24 \mathrm{~h}$. The excess of the amine and the solvent were evaporated off under vacuum and the crude polymer was dissolved in methanol. The solution was added to excess diethylether resulting in the precipitation of the pure polymer.

\section{Poly (vinylbenzyltriethylammonium chloride) (PVBTEAC)}

PVBC ( $1.0 \mathrm{~g}, 6.56 \mathrm{mmol})$ was treated with triethylamine ( $6 \mathrm{~mL}, 43 \mathrm{mmol}$ ) under the same conditions as above to obtain PVBTEAC. It was purified as described above.

\section{Poly(vinylbenzyltriphenylphosphonium chloride) (PVBTPPhC) $^{14}$}

The mixture of PVBC ( $2.0 \mathrm{~g}, 13.1 \mathrm{mmol})$ in DMF $(75 \mathrm{~mL})$ and triphenylphosphine ( $13.3 \mathrm{~g}, 50.8 \mathrm{mmol})$ were stirred at $80^{\circ} \mathrm{C}$ for $24 \mathrm{~h}$. The solution was concentrated and precipitated in diethylether. The product was dissolved in DMF and added to excess diethylether. The precipitated polymer was again dissolved in methanol and reprecipitated in excess of diethylether to get the pure polymer.

\section{Copolymers of polyvinylbenzylacetate (PAC) ${ }^{15}$ and PVBTEAC}

(a) To PVBC ( $0.61 \mathrm{~g}, 4 \mathrm{mmol}$ ) dissolved in toluene $(10 \mathrm{~mL})$, potassium acetate $(0.39 \mathrm{~g}, 4 \mathrm{mmol})$ and

Table I. The Structure of the Polycations Used in This Study

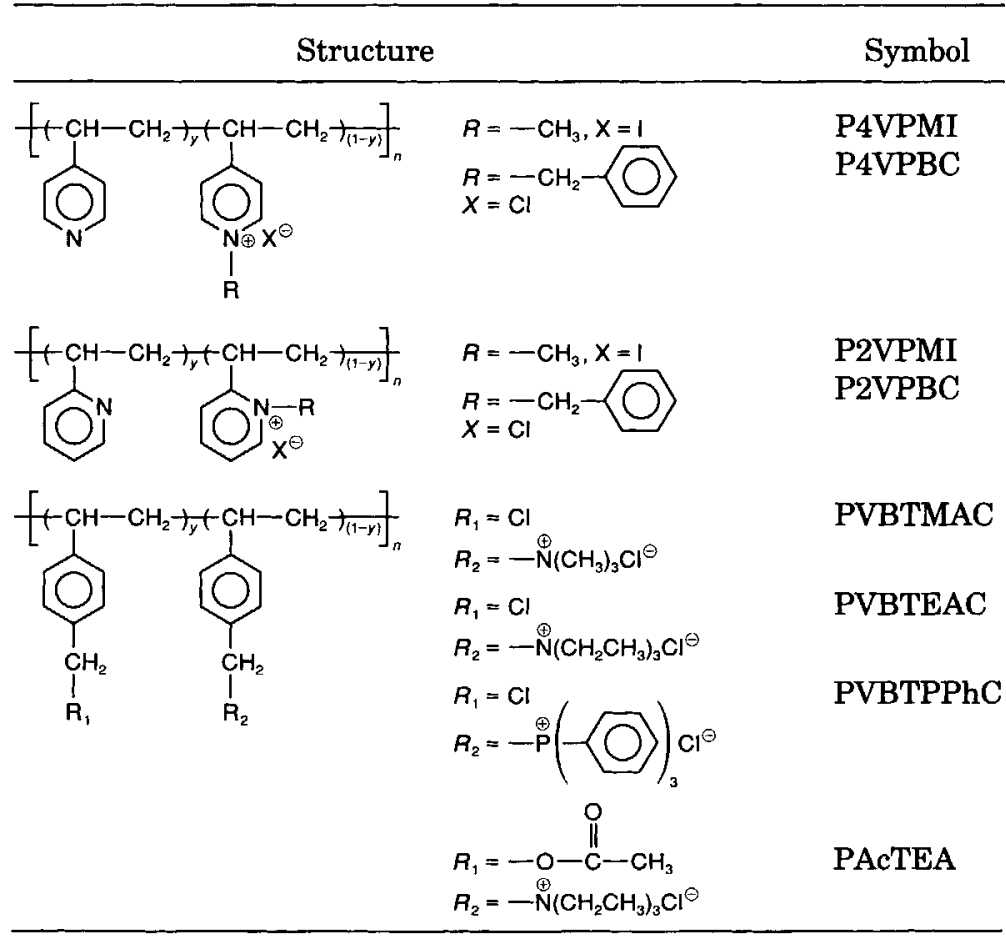


tetrabutylammonium bromide $(0.13 \mathrm{~g}, 0.4 \mathrm{mmol})$ were added. The reaction mixture was stirred at room temperature for $24 \mathrm{~h}$ and poured into methanol. The precipitated product was dissolved in THF and the solution was poured in excess of water to precipitate the polymer. The process was repeated twice and the resulting polymer precipitate was washed with methanol and dried. The degree of esterification of the product was 0.32 , as determined by the ${ }^{1} \mathrm{H}-\mathrm{NMR}\left(\mathrm{CDCl}_{3}\right): \delta=4.9\left(-\mathrm{CH}_{2}-\mathrm{O}-\right)$; $4.4\left(-\mathrm{CH}_{2}-\mathrm{Cl}\right)$. To the esterified product $(0.81$ $\mathrm{g})$ in dioxane $(12 \mathrm{~mL})$, triethylamine $(5 \mathrm{~mL}, 35.9$ $\mathrm{mmol}$ ) was added. The reaction mixture was stirred at room temperature for $24 \mathrm{~h}$ with the addition of water on appearance of turbidity. The product was separated by vacuum evaporation of the reagents. It was designated as PAcTEA-HDS.

(b) The mixture of PVBC $(2.0 \mathrm{~g}, 13.1 \mathrm{mmol})$ in toluene $(30 \mathrm{~mL})$, potassium acetate $(1.38 \mathrm{~g}, 14$ $\mathrm{mmol})$ and tetrabutylammonium bromide $(0.43 \mathrm{~g}$, $1.32 \mathrm{mmol}$ ) was treated as described above. In this case, the degree of esterification of the product was 0.65 . To the product polymer $(1.2 \mathrm{~g})$ in dioxane ( 35 $\mathrm{mL}$ ), triethylamine ( $4.1 \mathrm{~mL}, 29.4 \mathrm{mmol})$ was added and the mixture was stirred for $24 \mathrm{~h}$ at room temperature. The aminated product (designated as PAcTEA-LDS) was separated by the evaporation of the excess solvent and reagents, dissolved in methanol, and precipitated in excess of diethylether.

\section{Preparation of Quaternized Polyvinylpyridine Samples}

\section{Poly (4-vinylpyridiniumbenzyl chloride) ${ }^{16}$ of High DS (P4VPBC-HDS)}

Poly (4-vinylpyridine) (P4VP) ( $1.0 \mathrm{~g}, 9.52 \mathrm{mmol})$ and benzyl chloride ( $10 \mathrm{~mL}, 86.9 \mathrm{mmol})$ in nitromethane $(20 \mathrm{~mL})$, were stirred at room temperature for $48 \mathrm{~h}$. The product was separated by evaporation of the excess reagents under vacuum and was dissolved in ethanol. The pure polymer was precipitated from the solution in excess of toluene.

\section{P4VPBC of Very Low DS (P4VPBC-VLDS)}

P4VP ( $1.76 \mathrm{~g})$ in methanol $(35 \mathrm{~mL})$ was treated with benzyl chloride $(0.7 \mathrm{~mL}, 6.08 \mathrm{mmol})$ for $12 \mathrm{~h}$ at room temperature. The product was purified as above.

\section{Poly (2-vinylpyridiniumbenzyl chloride $)^{17}$ of Low DS (P2VPBC-LDS)}

Benzyl chloride ( $6 \mathrm{~mL}, 52.13 \mathrm{mmol}$ ) was added to poly (2-vinylpyridine) (P2VP) (1.0 g, $9.52 \mathrm{mmol}$ ) in nitromethane $(20 \mathrm{~mL})$. The mixture was stirred at $70^{\circ} \mathrm{C}$ for $6-7$ days. The product was separated as above, dissolved in ethanol, and poured into excess of toluene to obtain the pure polymer as a precipitate.

\section{Poly (2-vinylpyridiniumbenzyl chloride) of Very Low DS (P2VPBC-VLDS)}

Benzyl chloride $(3.8 \mathrm{~mL}, 33 \mathrm{mmol})$ was added to P2VP (1.0 g, $9.52 \mathrm{mmol})$ in methanol $(20 \mathrm{~mL})$ and stirred at room temperature for 2 days. The product was purified as described above.

All the compounds prepared were dried at $50^{\circ} \mathrm{C}$ under vacuum. The DS were determined by argentometric titration using amperometry. ${ }^{18}$ The molecular weight ( $\mathrm{mol} \mathrm{wt}$ ) and the DS of these compounds are listed in Table II.

The aqueous solutions of the polymers were made based on the molar concentration of the charged sites. For dye-binding experiments, a set of solutions was made with different ratios of the polymer and the dye. In all the solutions, the concentration of the dye was kept constant $\left(4.0 \times 10^{-5} \mathrm{M}\right)$ and the polycation concentration was changed (0.0-3.0 $\left.\times 10^{-4} \mathrm{M}\right)$. Upon equilibration, the UV-vis spectra were recorded (Hitachi U3400).

\section{RESULTS AND DISCUSSION}

The spectral behavior of $\mathrm{MO}$ in aqueous solution has been studied in detail. ${ }^{7}$ The dye, in dilute so-

Table II. The mol wt, DS, Wavelength of the Highest Energy Band, $\lambda$, and $r$ Values for the Various Polymers Used in This Study

\begin{tabular}{lcccc}
\hline \multicolumn{1}{c}{ Polymer } & $\begin{array}{c}\text { mol wt } \\
\times 10^{-4}\end{array}$ & DS & $\begin{array}{c}\lambda \\
(\mathrm{nm})\end{array}$ & $\begin{array}{c}r \\
\left(\mathrm{M}^{-1} \mathrm{Cm}^{-1}\right)\end{array}$ \\
\hline P2VPMI-LDS & 2.3 & 0.40 & 415 & 0.079 \\
P2VPMI-HDS & 2.3 & 0.71 & 384 & 0.133 \\
P4VPMI-HDS & 8.0 & 0.82 & 379 & 0.183 \\
P2VPBC-VLDS & 31.0 & 0.23 & 455 & - \\
P2VPBC-LDS & 13.6 & 0.41 & 408 & 0.081 \\
P4VPBC-HDS & 8.0 & 0.71 & 377 & 0.162 \\
P4VPBC-VLDS & 80.4 & $\mathbf{0 . 2 0}$ & 428 & 0.092 \\
PVBTMAC & $\mathrm{a}$ & $\mathbf{0 . 7 9}$ & 404 & 0.114 \\
PVBTEAC & $\mathrm{a}$ & 0.86 & 410 & 0.138 \\
PVBTPPhC & $\mathrm{a}$ & 1.0 & 430 & - \\
PAcTEA-HDS & a & $\mathbf{0 . 6 1}$ & 413 & - \\
PAcTEA-LDS & a & 0.37 & 419 & - \\
\hline
\end{tabular}

a $\cap_{\mathrm{sp} / \mathrm{C}}$ of $\mathrm{PVBC}=0.24 \mathrm{dL} / \mathrm{g}$ in benzene at $30^{\circ} \mathrm{C}$ measured at a concentration of $0.5 \mathrm{~g} / \mathrm{dL}$. 
lution $\left(10^{-3}-10^{-6} \mathrm{M}\right)$ is shown to have an absorption peak at $465 \mathrm{~nm}$ due to the isolated monomeric species. Increasing concentration of the dye brings about a blue shift, which is attributed to the oligomeric aggregates of the dye molecules. The higher the aggregate, the lower is the wavelength of the corresponding absorption band.

The aggregation or the stacking of the dye itself can be brought about at a lower concentration by the addition of the polymers with specific binding sites for the dye. It has been shown that, with the addition of increasing amounts of the highly ionized cationic polyelectrolytes, the spectra of MO show a gradual blue shift of the $465 \mathrm{~nm}$ band due to the stacking of the dye on the polymer backbone. A high degree of stacking may lead to the appearance of a well-defined peak at higher energy, [e.g., at $370 \mathrm{~nm}$ for MO and poly (3-methyl-1-vinylimidazolium bromide) system] ${ }^{4}$ On increasing the concentration of the polymer, the blue-shifted band gradually disappears and the $465-\mathrm{nm}$ band reappears due to the spreading out of the dye molecules along the polymer chain. This provides further proof for the explanation that the blue-shifted bands are due to stacking. The larger the polyelectrolyte concentration required to restore the $465-\mathrm{nm}$ band, the stronger is the cooperativity or the tendency of the dye to occupy the adjacent sites on the polymer. The various polyelectrolytes used in this study give information about the effect of the structural parameters on the dye-polymer interactions. The UV-Vis spectra of MO in the presence of various polycations studied are shown in Figures 1-4.
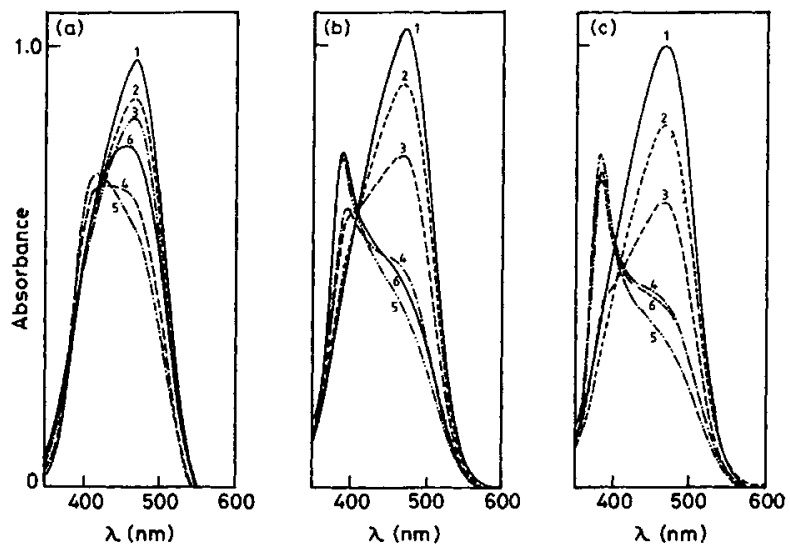

Figure 1. Absorption spectra of $\mathrm{MO}$ and methyl quaternized PVP cations at various $p$ : (a) P2VPMI-LDS: $p$ $=$ (1) 0.0, (2) 0.26, (3) 0.52, (4) 1.0, (5) 2.1, and (6) 7.2; (b) P2VPMI-HDS: $p=$ (1) 0.0 , (2) 0.25 , (3) 0.5, (4) 1.0, (5) 2.0 , and (6) 7.0; (c) P4VPMI-HDS: $p=$ (1) 0.0 , (2) 0.25 , (3) 0.51, (4) 1.0 , (5) 2.1 , and (6) 7.2 .
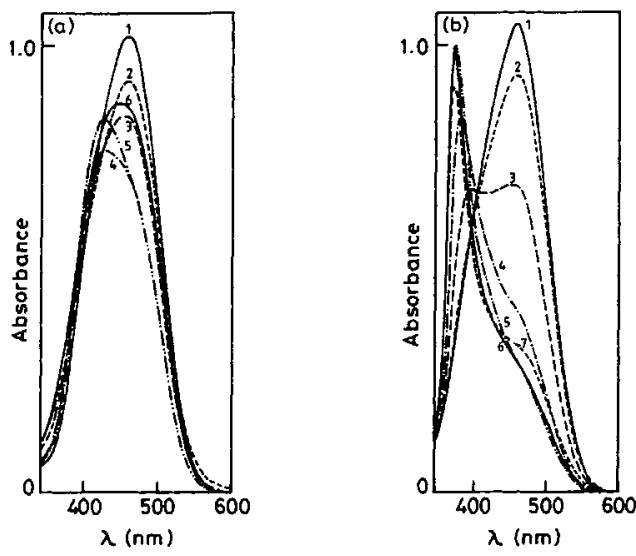

Figure 2. Absorption spectra of MO and benzyl quaternized PVP cations at various $p$ : (a) P4VPBC-VLDS and (b) P4VPBC-HDS: $p=$ (1) 0.0, (2) 0.25, (3) 0.50 , (4) 1.01, (5) $2.1,(6) 7.0$, and (7) 25.0 .

The binding process observed here can be explained by a simple theory ${ }^{19}$ of cooperative binding, which considers the cooperative interaction between the nearest neighboring binding sites. At low concentration, in the presence of the polymer, a dye can exist in the free and bound forms. In the bound form, the dye can experience two kinds of environments: the isolated bound states for which the neighboring sites are not occupied and the aggregated states in which the dye occupies a site in the immediate neighborhood of an already bound site. The molar extinction coefficient of the dye at $465 \mathrm{~nm}$ in such a system can be written as,

$$
\epsilon=\epsilon_{s t}+\left(\epsilon_{A}-\epsilon_{s t}\right)\left(\gamma_{A}+\xi_{A}\right) \rightarrow
$$
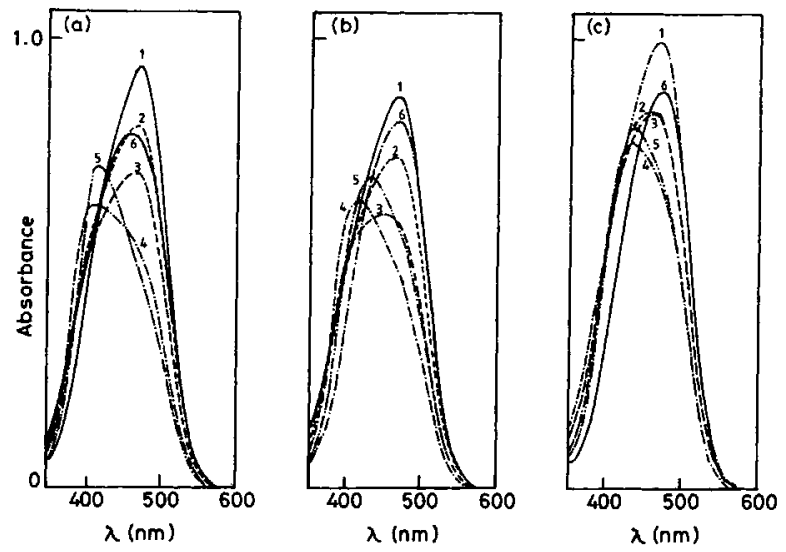

Figure 3. Absorption spectra for MO and PVBC derivatives at various $p$ : (a) PVBTMAC: $p=(1) 0.0$, (2) 0.27 , (3) 0.55 , (4) 1.10 , (5) 1.97 , and (6) 7.68 ; (b) PVBTEAC: $p=$ (1) 0.0 , (2) 0.29 , (3) 0.58 , (4) 1.17 , (5) 2.34, and (6) 8.18; (c) PVBTPPhC: $p=$ (1) 0.0, (2) 0.26, (3) 0.51, (4) 1.02, (5) 2.05 , and (6) 7.17 . 

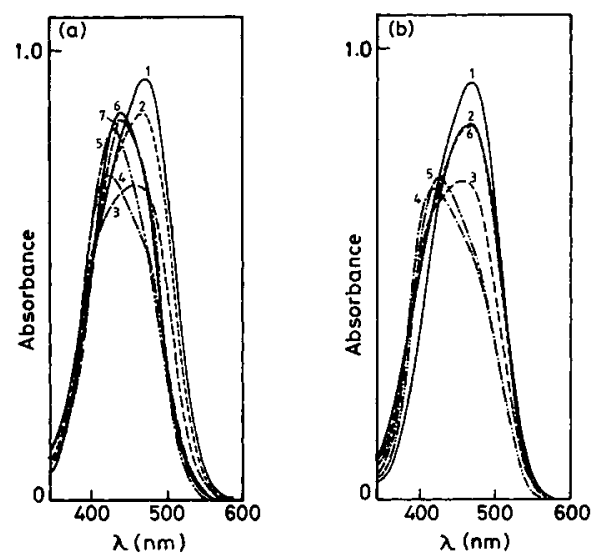

Figure 4. Absorption spectra for MO and PAcTEA: (a) PAcTEA-LDS: $p=$ (1) 0.0, (2) 0.28 , (3) 0.55, (4) 1.11, (5) 2.19 , (6) 7.74, and (7) 19.9; (b) PAcTEA-HDS: $p$ $=$ (1) 0.0, (2) 0.28 , (3) 0.56 , (4) 1.12 , (5) 2.23 , and (6) 7.81 .

where $\epsilon_{s t}$ and $\epsilon_{A}$ are the extiction coefficients at 465 $\mathrm{nm}$ of the stacked and the free unbound dye, respectively; and $\gamma_{A}$ and $\xi_{A}$ are the fractions of the free and the isolated bound dye, respectively. Strictly speaking, $\epsilon_{s t}$ is not a constant, as more than one species of oligomeric dye aggregates are present, which is evident from the nonexistence of a clear isosbestic point in the UV-Vis spectra. However, since the contribution of the stacked dye to the absorption at $465 \mathrm{~nm}$ is small, and the difference between the $\epsilon_{s t}$ of different oligomeric species is expected to be small, $\epsilon_{s t}$ has been assumed to be constant. For more accurate studies, the contribution of the monomeric species to the absorption at 465 $\mathrm{nm}$ can be deconvoluted from the spectra by curve fitting.

The fraction of the free dye can be expressed in terms of the fraction of the bound dye by the equation,

$$
\gamma_{A}=1-g \theta p \rightarrow
$$

where $\theta$ is the fraction of the occupied sites, $g$ is the number of binding sites per charged monomer segment of the polymer, and $p$ is the ratio of the molar concentration of the ionic groups of the polymer to that of the dye. Substitution of eq. (2) in eq. (1) yields

$$
\epsilon=\left(\epsilon_{s t}-\epsilon_{A}\right) g \theta p+\epsilon_{A}+\xi_{A}\left(\epsilon_{A}-\epsilon_{s t}\right) \rightarrow .
$$

The fraction of the occupied sites $\theta$ can be determined ${ }^{20}$ by evaluating the decrease of $\epsilon$ with increasing $p$. Our experimental data show that the plot of $\epsilon$ vs. $p$ is a straight line at small $p$ values with an intercept on the ordinate axis corresponding to $\epsilon_{A}$. The linear dependence of $\epsilon$ vs. $p$ implies that the last term in eq. (3) is negligible at low $p$ values; i.e., at small polymer-to-dye ratios, the concentration of the isolated bound dye is negligible and all the bound dye molecules are in the stacked form. The magnitude of the slope of the straight line given by the term $\left(\epsilon_{s t}-\epsilon_{A}\right) g \theta$ corresponds to the amount of stacking of the dye on the polymer and, hence, to the strength of the cooperative binding. The plot of $\epsilon$ vs. $p$ for the representative polymers studied here are given in Figure 5. The nature of the curves in Figures 5(a) $-5(\mathrm{c})$ and $5(\mathrm{e})$ are characteristic of a system where the aggregation of the bound dye molecules is favored over the isolated binding. The $\epsilon$ of the solution, which is a measure of the free dye, falls sharply at low $p$ values. After a certain value of $p$, the slope of the curve changes sharply, as the dye preferably binds on the isolated sites. The higher the value of $p$ at which the $\epsilon$ vs. $p$ curve deviates from linearity, the higher is the extent of cooperativity. In Figure 5(d), the slope of the $\epsilon$ vs. $p$ curve changes at very small $p$ values and in Figure 5(f), the curve is continuous without any break. Both these curves reflect a case of weak cooperative interaction in which the dye molecules take up isolated sites on the polymer even at low values of $p$. The extent of stacking, given by the slope $\left(\epsilon_{s t}-\epsilon_{A}\right) g \theta$ and represented as $r$, is listed in Table II for different systems. The dependence of $r$ on the structural parameters of the polymers is discussed below.

\section{Polyvinylpyridine (PVP) derivatives}

\section{The Effect of Charge Density on Dye Binding}

The spectral behavior of MO in the presence of P2VPMI of two different DS is shown in Figure 1.

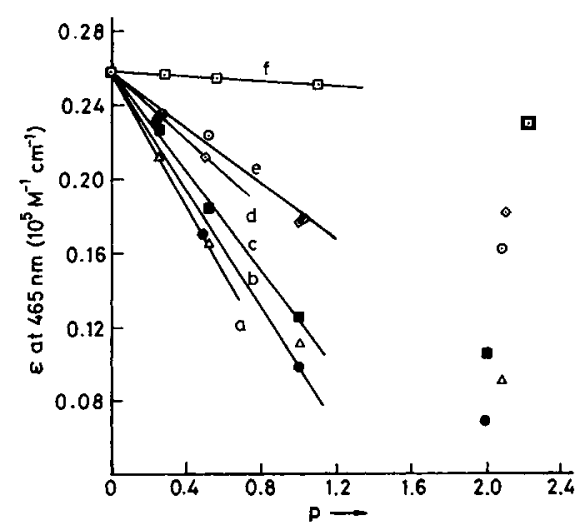

Figure 5. Plot of $\epsilon$ vs. $p$ for (a) P4VPMI-HDS, (b) P4VPBC-HDS, (c) P2VPMI-HDS, (d) P4VPBC-VLDS, (e) P2VPMI-LDS, and (f) P2VPBC-VLDS. 
The blue shift of the dye absorption band observed in both cases confirms the stacking of dye on both the polymers. In the case of P2VPMI of low DS, the blue shift of the dye absorption band at maximum stacking is only $50 \mathrm{~nm}$ [see Fig. 1(a)] and the absorption peak at $465 \mathrm{~nm}$ is restored at a $p$ of 7.2. For P2VPMI of high DS, the absorption of the stacked dye appears as a sharp peak at $384 \mathrm{~nm}$, corresponding to a larger shift of $80 \mathrm{~nm}$ [Fig. 1(b)]. The original $465 \mathrm{~nm}$ band is not restored even on adding a large excess of the polymer $(p=50.0)$. This is due to the higher degree of cooperativity in this case compared to the former. A similar behavior is shown by P4VPMI-HDS and P4VPBC-HDS also [Fig. 1(c) and 2(b)]. The higher value of $r$ (Table II) in the case of P2VPMI-HDS, P4VPMI-HDS, and P4VPBC-HDS compared to the corresponding LDS derivatives demonstrates a higher stacking of the dye on the polymers with high charge density due to the shorter distance between the binding sites on the polymer backbone.

\section{The Effect of the Nature of the Quaternizing Side Chains}

It is well known that in addition to coulombic forces, the nonionic interactions between the dye molecules and the polymer side chains contribute toward the binding mechanism. The benzyl and the hexyl quaternized polyvinylimidazole are reported ${ }^{4}$ to show enhanced binding strength relative to the methyl derivatives. In P4VP partially quaternized with methyl, ethyl, and propyl chains, the first binding constant for MO $\left(K_{1}\right)$ increased in the same order as the hydrophobicity of the side chains. ${ }^{9}$ We have investigated the effect of the alkyl and the aryl side chains on the dye-binding characteristics of the quaternized P2VP and P4VP. The spectral changes of $\mathrm{MO}$ in the presence of P4VPBC (VLDS and HDS) are shown in Figures 2(a) and 2(b). Comparison of Figures 2(b) and 1(c) shows that the aggregation behavior of the dye is similar for the methyl and the benzyl quaternized P4VP derivatives of high charge density. The corresponding plots in Figure 5 [ see (a) and (b)] show nearly equal slope. The deviation of the curves from the straight line nature occurs at a lower value of $p$ for the methyl quaternized P4VP. This indicates that the binding on isolated sites is stronger in this case, compared to the benzyl derivative, which could be due to the lesser steric hindrance offered by the methyl group. But, at low $p$ values, the steric hindrance of the benzyl derivatives is nearly, though not fully, compensated by the involvement of the $\pi$ electrons of the benzyl group in the stacking process, resulting in nearly equal extent of stacking on both the polymers. A similar effect of an increase in the interaction energy between the dye and the polymer due to the intercalation of the dye between the side chain rings in the polymer has been reported for MO and poly (3benzyl 1-vinylimidazolium bromide) ${ }^{3}$ The low DS polymers (P2VPMI-LDS and P2VPBC-LDS) show identical behavior regarding the amount of stacking (Table II) and the extent of cooperativity, which is probably due to the absence of steric hindrance, as the binding sites are well separated from each other.

\section{The Effect of the Location of the Binding Site}

It is evident from the higher value of $r$ for P4VP derivatives (Table II) that the stacking of the dye on these polymers is much more than on the P2VP derivatives, which is due to the difference in steric hindrance offered by the binding sites in the two cases. However, for the same reason, the binding constant for the isolated sites is also higher in the case of $\mathrm{P} 4 \mathrm{VP}$ derivatives, resulting in the deviation of the $\epsilon$ vs. $p$ curve from linearity at relatively lower values of $p$ in these polymers [Figs. $5(a)$ and (d)]. Thus, the easy availability of the charged site on the polymer chain seems to be an important factor for the favorable binding of the dye.

\section{Quaternized PVBC Derivatives}

\section{The Effect of the Nature of the Quaternizing Side Chain}

Aggregation of dye molecules on the PVBTEAC and the blue shift of the $465-\mathrm{nm}$ band have been reported by Quadrifolio and Crescenzi. ${ }^{7}$ We have carried out similar studies with PVBTEAC and compared the results with trimethylamine and triphenylphosphine derivatives. The absorption spectra of the dye in the presence of the above polymers is shown in Figure 3 . The absorption for the highest form of the stacked dye, i.e., the maximum blue-shifted peak, appears at 404,413 , and $430 \mathrm{~nm}$ for the trimethylamine, triethylamine, and triphenylphosphine derivatives, respectively. The smaller magnitude of the blue shift of the 465-nm peak indicates low extent of stacking on these polymers compared to the PVP derivatives. The extent of stacking $(r)$ has been calculated from the slope of the $\epsilon$ vs. $p$ curves (not shown) for the trialkylammonium derivatives. The corresponding plot for the PVBTPPhC shows a continuous change in slope, because of which the $r$ value could not be calculated. From the initial slope of $\epsilon$ vs. $p$ curves, the stacking tendency of MO seems to be similar on 
these polymers irrespective of the nature of the quaternizing side chain. The extent of restoration of the 465-nm peak at $p=7-8$ is in the order PVBTMAC < PVBTEAC < PVBTPPhC, which shows that the binding strength of the dye at the isolated sites follows the same order as the hydrophobicity of the quaternizing side chain. The nonpolar interaction between the pendant groups of the polycations and the dye seems to increase the binding strength of the dye at the isolated sites of these polymers.

\section{The Effect of Charge Density on Dye Binding}

The P4VBC derivatives of low DS tend to form ether crosslinks on reacting with water. In order to make soluble quaternary salts of PVBC of various DS, a certain number of the chloromethyl groups were initially blocked by esterification and the remaining were used for the quaternization reaction. The dyebinding characteristics of the PAcTEA derivatives, esterified and aminated to different extent, are shown in Figure 4. In the case of PAcTEA-HDS, the absorption peak for the highest form of the stacked dye appears at a wavelength $(413 \mathrm{~nm})$ that is very close to that of PVBTEAC (410 nm), showing similar stacking on both the polymers in spite of the reduction in charge density of the former. Further, in the case of PAcTEA-LDS, where the charge density is reduced to half, the blue shift $(419 \mathrm{~nm})$ is not much less compared to the PAcTEA-HDS, and the $465-\mathrm{nm}$ peak is not restored even at a large $p$ value $(p=20.0)$. This shows the involvement of the ester groups in the enhancement of stacking of the dye. This enhancement of stacking in spite of the large decrease in the electrostatic interaction is unexpected. However, it is known that nonionic forces between the dye and the polymer side chain do enhance the dye binding. ${ }^{4,6}$ More elaborate studies are to be carried out in this regard.

\section{CONCLUSIONS}

The dye-binding ability of the polycations is observed to have a strong dependence on the structure of the polymer. It is favored by the easy availability of the charged site on the polymer. The stacking tendency of the dye is enhanced by the high charge density of the polymer. The binding ability of the PVP-based polycations is higher than the quater- nized PVBC polymers. The loss of cooperative binding at high $p$ values and the preference for isolated sites on the polymer is dependent on the strength of the binding of the dye at isolated sites.

The author gratefully acknowledges S. Ghosh for helpful discussions and V. Kalpagam for constant encouragement.

\section{REFERENCES AND NOTES}

1. I. M. Klotz, Adv. Chem. Phys., 39, 109 (1978).

2. T. Takagishi, T. Susimoto, A. Hayashi, and N. Kuroki, J. Polym. Sci., Polym. Chem. Ed., 21, 2311 (1983).

3. B. J. Orchard, J. S. Tan, and A. J. Hopfinger, Macromolecules, 17, 169 (1984).

4. T. M. Handel, H. L. Cohen, and J. S. Tan, Macromolecules, 18, 1200 (1985).

5. W. Dawydoff, K. J. Linow, and B. Philipp, Acta Polymerica, 42, 592 (1991).

6. I. M. Klotz, G. P. Roger, and A. R. Slomewsky, Biochemistry, 8, 4752 (1969).

7. F. Quadrifoglio and V. Crescenzi, J. Colloid. Interface. Sci., 35, 447 (1971).

8. K. Ishizu, Y. Kashi, T. Fukutami, and T. Kakmai, Makromol. Chemie., 183, 3099 (1982).

9. W. S. Kim, K. H. Seo, and Y. J. Lim, J. Polym. Sci., Polym. Chem. Ed., 27, 389 (1989).

10. T. Takagishi, K. Yoshikawa, H. Hamano, N. Kuroki, and H. Kozuka, J. Polym. Sci., Polym. Chem. Ed., 23, 37 (1985).

11. T. Takagishi, Y. Nataka, and N. Kuroki, J. Polym. Sci., Polym. Chem. Ed., 12, 807 (1974).

12. B. Vishalakshi, S. Ghosh, and V. Kalpagam, Polymer, 34, 3270 ( 1993).

13. G. D. Jones, Ind. Eng. Chem., 44, 2686 (1952).

14. P. Hodge, B. J. Hunt, E. Koshdel, and J. Waterhouse, Polym. Prepr., 23, 147 (1982).

15. T. Lizawa, T. Nashikuba, and Y. Masuda, Macromolecules, 17, 992 (1984).

16. T. Okubo and N. Ise, J. Amer. Chem. Soc., 95, 2293 (1973).

17. S. Forster, M. Schmidt, and M. Antonietti, Polymer, 31, 783 (1990).

18. The Principles and Applications of Polarography, G. W. C. Milner, Ed., Longmans, London, 1962, p. 691.

19. G. Schwarz, Eur. J. Biochem., 12, 442 (1970).

20. G. Schwarz, S. Klose, and W. Balthasar, Eur. J. Biochem., 12, 454 (1970).

Received March 3, 1994

Accepted August 6, 1994 